\title{
X-Ray Peak Profile Analysis of Nanostructured Hydroxyapatite and Fluorapatite
}

\author{
K. Venkateswarlu, D. Sreekanth, M. Sandhyarani, V. Muthupandi, A. C. Bose, and N. Rameshbabu
}

\begin{abstract}
In present study, X-ray peak profile analysis (XPPA) by modified Williamson-Hall (W-H) models, namely W-H-isotropic strain model (W-H-ISM), W-H-anisotropic strain model (W-H-ASM) and W-H-energy density model (W-H-EDM), was employed to estimate the microstructural parameters such as, crystallite size, lattice strain, lattice deformation stress and deformation energy density from the powder diffraction data obtained for the microwave synthesized hydroxyapatite (HA) and fluorapatite (FA) nanoparticles prepared under identical processing conditions of mixing and aging. The as-prepared powder particles were also characterized by transmission electron microscopy (TEM) method. The average crystallite size values estimated for HA and FA by XPPA were correlated to their respective transmission electron microscopy (TEM) analysis results. In addition, the estimated values of $\mathrm{HA}$ and $\mathrm{FA}$ were correlated to their in-vitro dissolution characteristics studied by ethylenediamine tetra-acetic acid (EDTA) titrimetric method. It is found that the average crystallite size estimated by $\mathrm{W}-\mathrm{H}$ models is in good agreement with TEM results. The controlled in-vitro dissolution behavior of FA was found to be resulted out of its higher crystallite size, lower lattice strain and lower dislocation density compared to that of HA.
\end{abstract}

Index Terms-Hydroxyapatite, fluorapatite, biomedical applications, defects, $\mathrm{X}$-ray peak profile analysis.

\section{INTRODUCTION}

Synthetic hydroxyapatite $\left[\mathrm{HA} ; \mathrm{Ca}_{10}\left(\mathrm{PO}_{4}\right)_{6}(\mathrm{OH})_{2}\right]$ has been extensively used as an implant material, because of its similarity with human bone composition and thereby its ability to form a strong bond to the human hard tissue. However, synthetic HA presents poor thermal stability. On the other hand, fluorapatite $\left.\left[\mathrm{FA} ; \mathrm{Ca}_{10}\left(\mathrm{PO}_{4}\right)_{6} \mathrm{~F}_{2}\right)\right]$ exhibits lower dissolution rate and high thermal stability by retaining its phase purity up to a temperature of $1400{ }^{\circ} \mathrm{C}$. Further, FA is found to form as an outer layer in tooth enamel [1]. Hence, FA is desirable as an outer layer in tooth enamel, because it is less soluble in acidic solutions (like those produced by oral bacteria or by Diet Coke) than the HA.

Many researchers have addressed the influence of fluorine

Manuscript received July 24, 2012; revised October 23, 2012.

K. Venkateswarlu is with the Department of Physics and Department of Metallurgical and Materials Engineering, National Institute of Technology, Tiruchirappalli, 620015, Tamil Nadu, India (e-mail: kotharu.venkat@ gmail.com).

A. C. Bose is with the Department of Physics, National Institute of Technology, Tiruchirappalli, 620015, Tamil Nadu, India (e-mail: acbose@nitt.edu)

D. Sreekanth, M. Sandhya Rani, V. Muthupandi, and N. Rameshbabu are with the Department of Metallurgical and Materials Engineering, National Institute of Technology, Tiruchirappalli, 620015, Tamil Nadu, India (e-mail: sreekanth54@gmail.com, muthyala07@gmail.com, vmuthu@nitt.edu, rameshrohitht@gmail.com).
(F) incorporation within apatite lattice on the biological, mechanical and physicochemical properties of HA such as, thermal stability [2], solubility [3], stem cell growth-colonisation [4], hardness, crystallinity and corrosion resistance [5]-[7]. Thus most of the existed studies focussed on qualitative description of the variations in the properties shown by HA and F-HA. But a certain gap exists in the literatures to quantify the microstructural parameters for better understanding of the observed differences in physicochemical properties of FA compared to HA.

Nanocrystalline HA is expected to have homogeneous resorption and better bioactivity than coarser crystals [8]. Further, the properties shown by nanomaterials are crystallite size dependant. Thus, for complete understanding of nanoscale material properties, the crystallite size and other related microstructural quantities such as dislocation density need to be calculated correctly.

Keeping in mind the need and importance of analytical methods such as X-ray peak profile analysis (XPPA) to estimate the microstructural quantities and to correlate them to the observed material properties, the present work aims at estimating the crystallite size, lattice strain, lattice stress and lattice strain energy density of $\mathrm{HA}$ and FA, based on conventional W-H plot by using W-H-ISM, W-H-ASM and W-H-EDM models, thereby correlating them to the differences in the literature reported and presently observed physicochemical properties of nanocrystalline HA and FA.

\section{MATERIALS AND METHODS}

\section{A. Synthesis and Characterization of HA and FA Nanoparticles}

The precursors used and the detailed procedure for the synthesis of HA [9] and FA [10] particles was reported in one of the authors previous study.

The X-ray diffraction (XRD) and transmission electron microscopy (TEM) techniques were employed to study the phase composition, the size and the morphology of as-prepared HA and FA powders. The isolated and preferred high intensity diffraction peaks namely, (002), (102), (202), (311), (222), (213) and (004) were chosen to estimate the microstructural parameters by various models based on $\mathrm{W}-\mathrm{H}$ plot.

\section{B. In-Vitro Dissolution Study}

The dissolution behaviour of the as-prepared HA and FA powder particles was studied by determining the amount of calcium released from each of the samples into the host ambience (4.5 $\mathrm{pH}$ medium) by EDTA titrimetric method. 
cylindrical glass bottle with $4.5 \mathrm{pH}$ solution and maintained at temperature $37{ }^{\circ} \mathrm{C}$ for $72 \mathrm{~h}$. The $\mathrm{pH}$ was maintained throughout $72 \mathrm{~h}$ by adding variable quantity of $0.001 \mathrm{~N}$ $\mathrm{HNO}_{3}$. After $72 \mathrm{~h}$ the supernatant liquid was collected by micro pipette and centrifuged at $3500 \mathrm{rpm}$ for 15 minutes. 1 $\mathrm{ml}$ of $1 \mathrm{~N} \mathrm{NaOH}$ was added to $10 \mathrm{ml}$ of sample taken in a conical flask. A pinch of ammonium purpurate powder followed by $\mathrm{NaOH}$ solution was added to the above solution which ends up the solution with pink colour. This solution in the conical flask was titrated against $0.02 \mathrm{~N}$ EDTA solution taken in the burette and the titration reaction was carried out till the colour of the solution changes from pink to purple. The volume of EDTA consumed was noted. The amount of calcium released was determined by the following formulae [11]. Amount of calcium (P) present in the given sample in terms of $\mathrm{CaCO}_{3}$

$$
P=\frac{\text { volume of } E D T A \times 1000}{\text { volume of sample taken }}
$$

Calcium present in the sample $(\mathrm{mg} / \mathrm{l})=$

$$
P \times \frac{\text { Molecular weight of calcium }}{\text { Molecular weight of calcium carbonate }}
$$

\section{RESULTS AND DISCUSSION}

\section{A. XRD Analysis of HA and FA Nanoparticles}

The XRD patterns of HA and FA samples are shown in Fig. 1(a) and 1(b) respectively.

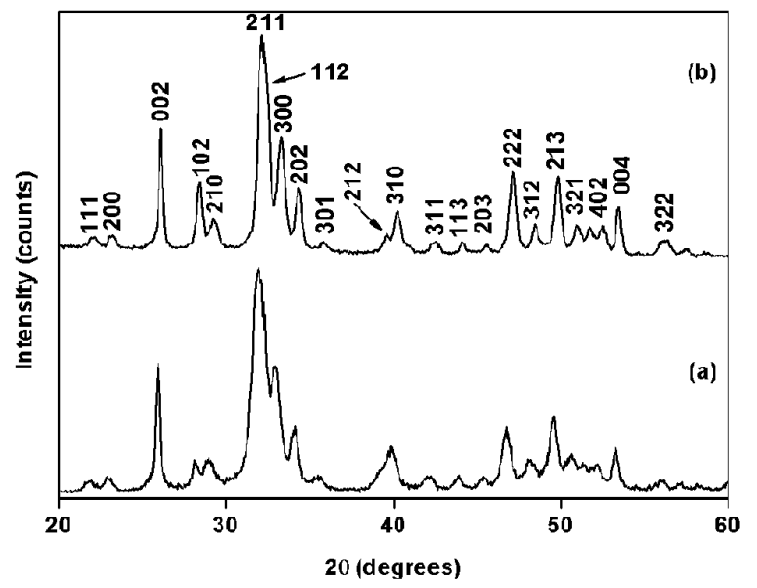

Fig. 1. XRD patterns of as-prepared nanosized HA (a) and FA (b).

The XRD patterns of HA and FA are similar and exhibit several diffraction peaks which can be assigned to hexagonal crystal structure with space group P63/m. In addition, the diffraction peaks are markedly broader suggesting that the prepared powder particles were in nanosize. As compared to the broad X-ray diffraction peaks of HA particles in [Fig. 1 (a)], the diffraction peaks of FA particles [Fig. 1 (b)] are narrow and well separated. It confirms that FA sample is of improved crystallinity and increased crystallite size. The calculated percentage crystallinity of HA and FA are 22 and 50. The improved crystallinity of FA may come from the driving force provided by the high affinity $\mathrm{F}^{-}$groups (compared to $\mathrm{OH}^{-}$groups in the precursor solution) for the apatite crystal growth during precipitation [10].

\section{B. Determination of Crystallite Size of HA and FA by Scherrer Analysis}

The instrumental corrected broadening $\beta_{h k l}$ [12] corresponding to the diffraction peaks of HA and FA was estimated using the equation

$$
\beta_{h k l}=\left[\left(\beta_{h k l}\right)_{\text {measured }}-\left(\beta_{h k l}\right)_{\text {instrumental }}\right]^{1 / 2}
$$

Here the crystallite size value is calculated by using Scherrer's formula to compare with the values obtained from the W-H models and TEM analysis results. The crystallite sizes $D_{v}$ of HA and FA were estimated from the Scherrer's equation as given below:

$$
D_{v}=\frac{k \lambda}{\beta_{h k l} \cos \theta_{h k l}}
$$

where $D_{v}$ is the volume weighted crystallite size $(\mathrm{nm}) ; k$ is the shape factor $(k=0.9) ; \lambda$ is the wave length of the X-rays $(\lambda=$ $0.154056 \mathrm{~nm}$ for $\mathrm{Cu} K \alpha_{1}$ radiation); $\theta_{h k l}$ is Bragg diffraction angle $\left(^{\circ}\right)$ and $\beta_{h k l}$ is the broadening of the $h k l$ diffraction peak measured at half of its maximum intensity (in radians).

C. Estimation of Lattice Strain ( $\varepsilon$ ) and Crystallite Size (Dv) by $W-H-I S M$

The XPPA by W-H models is a simplified method which clearly differentiates between size induced and strain induced peak broadening by considering the peak width as a function of $2 \theta$ [13].
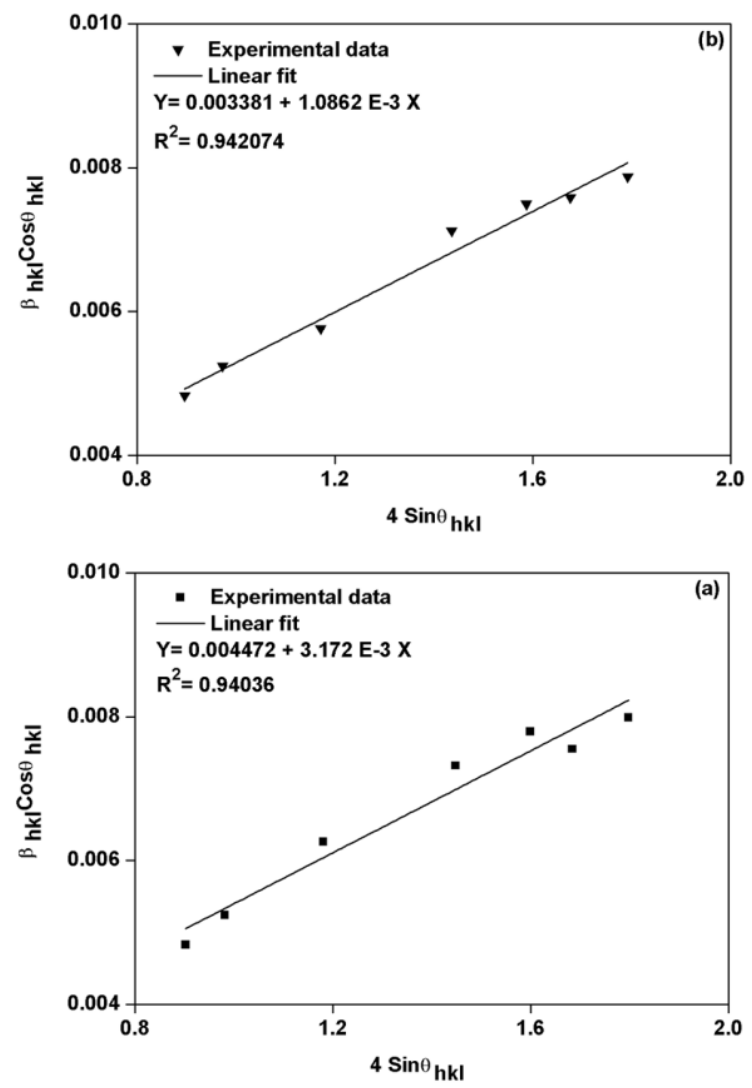

Fig. 2. W-H-ISM plots of as-prepared nanosized HA (a) and FA (b)

The strain induced line broadening $\beta_{s}$ is given by the relation $\beta_{s}=4 \varepsilon \tan \theta_{h k l}$. Now the total peak broadening is represented by the sum of the contributions of crystallite size and strain present in the material. 
TABLE I: THE ESTIMATED MicrostruCturaL PARAMETERS OF AS-PREPARED NANOSIZED HA AND FA.

\begin{tabular}{|c|c|c|c|c|}
\hline Analysis & Model & Parameter (unit) & $\begin{array}{l}\text { Estima } \\
\text { HA }\end{array}$ & FA \\
\hline \multirow[t]{4}{*}{ Scherrer analysis } & & $D_{v}(\mathrm{~nm})$ & 20 & 29 \\
\hline & & $D_{v}(\mathrm{~nm})$ & 31 & 42 \\
\hline & W-H-ISM & $\varepsilon \times 10^{-3}$ (no unit) & 3.172 & 1.086 \\
\hline & & $D_{v}(\mathrm{~nm})$ & 28 & 38 \\
\hline \multirow[t]{5}{*}{ W-H analysis } & W-H-ASM & $\sigma(\mathrm{MPa})$ & 64 & 41 \\
\hline & & $\varepsilon \times 10^{-3}$ (no unit) & 0.457 & 0.294 \\
\hline & & $D_{v}(\mathrm{~nm})$ & 30 & 40 \\
\hline & W-H-EDM & $\mathrm{u}\left(\mathrm{kJm}^{-3}\right)$ & 51 & 40 \\
\hline & & $\varepsilon \times 10^{-3}$ (no unit) & 0.853 & 0.758 \\
\hline TEM analysis & & $D_{v}(\mathrm{~nm})$ & $28 \pm 5$ & $39 \pm 6$ \\
\hline
\end{tabular}

Assuming that the strain present in HA and FA crystals is isotropic, the W-H equation [12] for the total peak broadening is given by

$$
\beta_{h k l} \cos \theta_{h k l}=\frac{k \lambda}{D_{v}}+4 \varepsilon \sin \theta_{h k l}
$$

A plot is drawn for HA and FA by taking $4 \sin \theta_{\mathrm{hk}}$ along $\mathrm{X}$-axis and $\beta_{h k l} \cos \theta_{h k l}$ along Y-axis as shown in Fig. 2 (a) and (b), respectively. In W-H-ISM model, the lattice strain values and the crystallite size values of HA and FA are respectively extracted from the slopes and the intercepts of the respective linear fits made to their experimental data. The estimated values of the lattice strain and the crystallite size for HA and FA are shown in Table I.

\section{Estimation of Lattice Deformation Stress $(\sigma)$ and Crystallite Size (Dv) by W-H-ASM}

According to Hook's law, the relation between stress and strain is given by $\sigma=E \varepsilon$, where $\mathrm{E}$ is the modulus of elasticity or Young's modulus. Assuming a small strain to be present in HA and FA particles, Hook's law can be applied to estimate the lattice deformation stress to a reasonable approximation. Hence by assuming that the lattice deformation stress is uniform in all crystallographic directions, the strain factor in second term of the equation (3) is replaced by $\varepsilon=\sigma / E_{h k l}$ and the modified equation is given by

$$
\beta_{h k l} \cos \theta_{h k l}=\frac{k \lambda}{D_{v}}+4 \sigma \sin \theta_{h k l} / E_{h k l}
$$

Here $E_{h k l}$ is Young's modulus in the direction normal to the set of (hkl) crystal lattice planes.

The crystallographic direction dependent Young's modulus in hexagonal crystals [14] of HA and FA is given by the following equation (5)

$$
E_{h k 1}=\frac{\left[h^{2}+\frac{(h+2 k)^{2}}{3}+\left(\frac{a_{1}}{c}\right)^{2}\right]^{2}}{s_{11}\left(h^{2}+\frac{(h+2 k)^{2}}{3}\right)^{2}+s_{33}\left(\frac{a_{1}}{c}\right)^{4}+\left(2 s_{13}+s_{44}\right)\left(h^{2}+\frac{(h+2 k)^{2}}{3}\right)\left(\frac{a_{1}}{c}\right)^{2}}
$$

Here $s_{11}, s_{33}, s_{44}$ and $s_{13}$ are the elastic compliances which are common for HA and FA and their standard hand book values are $7.49 \times 10^{-12}, 10.9 \times 10^{-12}, 15.1 \times 10^{-12},-4.0 \times 10^{-12}$, respectively [15]. The lattice deformation stress and crystallite size were respectively extracted from the slope and intersection of the HA and FA linear fits made to their respective $4 \sin \theta_{h k l} / E_{h k l}$ vs. $\beta_{h k l} \cos \theta_{h k l}$ plots. The estimated values of the lattice stress and the crystallite size values for HA and FA are shown in Table I.

\section{E. Estimation of Deformation Energy Density and Crystallite Size (Dv) by W-H-EDM}

In equation 3 , it is considered the homogeneous isotropic nature of the HA and FA crystals which, in many cases, is not fulfilled. Moreover, all the constants of proportionality associated with the stress-strain relation are no longer independent when the lattice strain energy density $u$ is considered. According to Hook's law $u=\varepsilon^{2} E_{h k l} / 2$ and therefore, the modified W-H-ASM equations for HA and FA can be expressed as

$$
\beta_{h k l} \cos \theta_{h k l}=\frac{k \lambda}{D_{v}}+4\left[\sin \theta_{h k l}(2 / E h k l)^{1 / 2}\right] u^{1 / 2} . .
$$

The lattice deformation energy density ' $u$ ' and the crystallite size values are separately extracted for HA and FA from the slope and the intersection of the linear fits made to their respective $\beta_{h k l} \cos \theta_{h k l}$ vs. $4 \sin \theta_{h k l}\left(2 / E_{h k l}\right)^{1 / 2}$ plots. The estimated crystallite size and lattice deformation energy density for HA and FA were shown in Table I.

\section{F. Estimation of Dislocation Density $(\rho)$}

Dislocation density is defined as the length of dislocation line per unit volume of the crystal. The dislocation density is calculated for HA and FA using the following relation [16].

$$
\rho=\frac{(3 n C / F)^{1 / 2}\left\langle e^{2}\right\rangle^{1 / 2}}{b D_{v}}
$$

where $n$, the number of dislocations on each face of the particle, $C$ is the constant depending on the strain distribution, $F$ is an interaction parameter, $\left\langle e^{2}\right\rangle^{1 / 2}$ is the root mean squared (RMS) strain, b is the burgers vector and $D_{v}$ is the crystallite size. For Cauchy strain profiles the value of $C$ is about 25, whereas for Gaussian strain profiles it is nearly 4. In the absence of extensive polygonization, dislocation density can be calculated from the above equation (4), by assuming $n \approx F$, $b=d$, the interplanar spacing and $C=4$. Now the equation (4) reduces to

$$
\rho=\frac{(12)^{1 / 2}\left\langle e^{2}\right\rangle^{1 / 2}}{d D_{v}}
$$


The estimated values of the dislocation density are $4.24 \times 10^{14}$ and $2.72 \times 10^{14}$ lines $/ \mathrm{m}^{2}$ respectively for HA and FA.

\section{G. In-Vitro Dissolution Study of HA and FA Particles}

The content of Ca released out of HA and FA into the test medium, as determined by EDTA titrimetric method, is 12 $\mathrm{mg} / \mathrm{l}$ and $8 \mathrm{mg} / \mathrm{l}$ respectively for $\mathrm{HA}$ and FA. It can be observed that the released $\mathrm{Ca}$ content is higher for HA compared to that of FA. This is due to the lower crystallinity, higher internal lattice strain and lower chemical stability of HA. Thus the higher solubility of HA can be attributed to its higher dislocation density compared to that of FA. Higher the dislocation density more is the dissolution rate. The effect of dislocation density on the dissolution behaviour of HA was explained in detail by [17]. Hence, it can be concluded that the HA with a higher dislocation density is more likely to be attacked by the acids on the surface, compared to that of FA. This results in the higher dissolution rate for HA compared to that of FA.

\section{H. TEM Analysis of HA and FA Particles}

The bright field transmission electron microscopic images of HA and FA nanoparticles in as-prepared condition were shown in Fig. 3 (a) and (b), respectively. The HA particles were of ellipsoid like morphology and were a bit shorter with more irregular and less clear contour. On the other hand, FA particles were a bit longer than HA particles with rod like morphology and with clear contours and less agglomeration. The sizes of HA and FA nanoparticles were estimated from TEM images by image analysis done with a freely available IMAGE J software. The HA particles exhibited a length of about $45 \mathrm{~nm}$ and a width of about $11 \mathrm{~nm}$ corresponding to an aspect ratio of about 4 . On the other hand the FA particles exhibited a length of about $65 \mathrm{~nm}$ and a width of about $13 \mathrm{~nm}$ corresponding to an aspect ratio of about 5. However, to correlate the results obtained from various analyses, the sizes of HA and FA obtained from TEM analysis were expressed as $($ length + width $) / 2$ and were given as $28 \pm 5 \mathrm{~nm}$ and $39 \pm 6 \mathrm{~nm}$ (mean $\pm \mathrm{SD}$ ) for HA and FA respectively.

\section{CONCLUSION}

XPPA was successfully employed to estimate the microstructural parameters of HA and FA powder particles. There is a good agreement between the crystallite size values obtained by $\mathrm{W}-\mathrm{H}$ analysis and that obtained by TEM analysis for HA and FA powder particles. Hence, $\mathrm{W}-\mathrm{H}$ analysis results are more accurate for the estimation of crystallite size, when compared to Scherrer analysis. Therefore it is suggested that the contribution from instrumental effects and lattice strain effect to the X-ray peak broadening need to be considered while calculating the crystallite size value of nanomaterials.

Within the materials elastic limit, the W-H models can be applied to estimate other microstructural parameters such as lattice strain, lattice deformation stress and lattice deformation energy density in a reasonable approximation. The crystallite size and the lattice strain values obtained from the W-H-ISM model for HA and FA can be further extended to estimate the dislocation densities present in the materials with more accuracy. The incorporation of $\mathrm{F}$ in the crystal lattice of HA results in higher crystallinity, crystallite size and reduced lattice strain, lattice stress, lattice strain energy density and dissolution rate of FA.
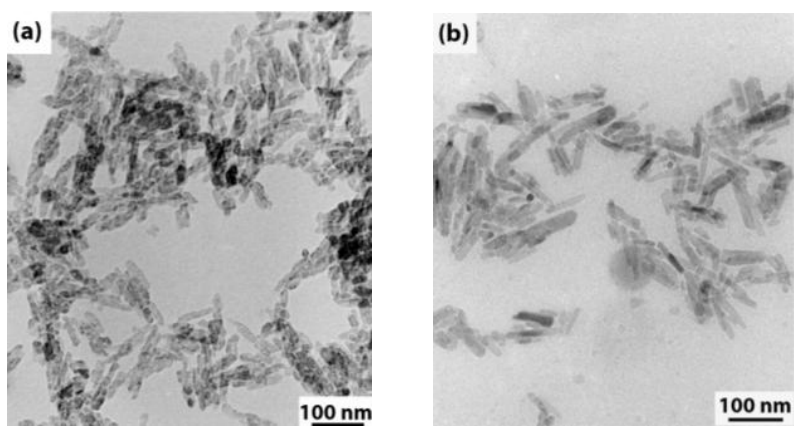

Fig. 3. TEM micrographs of as-prepared nanosized (a) HA and (b) FA.

\section{ACKNOWLEDGEMENT}

The authors would like to acknowledge the grants from Department of Biotechnology, New Delhi (BT/PR-11731/ MED/32/99/2008, dated 19-08-2009) and the Department of Science and Technology, New Delhi (SR/NM/NS-27/2008, dated 25-02-2009).

\section{REFERENCES}

[1] F. Brudevold, D. E. Gardner, and F. A. Smith, "The distribution of fluoride in human enamel," J. Dent. Res., vol. 35, pp. 420-429, 1956.

[2] A. Bianco, I. Cacciotti, M. Lombardi, L. Montanaro, E. Bemporad, and M. Sebastiani, "F-substituted hydroxyapatite nanopowders: Thermal stability, sintering behaviour and mechanical properties," Ceram. Int., vol. 36, pp. 313-322, 2010.

[3] M. Okazaki, Y. Miake, H. Tohda, T. Yanagisawa, T. Matsumoto, and J. Takahashi, "Functionally graded fluoridated apatites," Biomaterials, vol. 20, pp. 1421-1426, 1999.

[4] J. Harrison, A. J. Melville, J. S. Forsythe, B. C. Muddle, A. O. Trounson, K. A. Gross, and R. Mollard, "Sintered hydroxyfluorapatites-IV: The effect of fluoride substitutions upon colonisation of hydroxyapatites by mouse embryonic stem cells," Biomaterials, vol. 25, pp. 4977-4986, 2004.

[5] H. Qu and M. Wei, "The effect of fluoride contents in fluoridated hydroxyapatite," Acta Biomater., vol. 2, pp. 113-119, 2006.

[6] Y. Chen and X. Miao, "Effect of fluorine addition on the corrosion resistance of hydroxyapatite ceramics," Ceram. Int., vol. 30, pp. 1961-1965, 2004.

[7] S. J. Kim, H. G. Bang, J. H. Song, and S. Y. Park, "Effect of fluoride additive on the mechanical properties of hydroxyapatite/alumina composites," Ceram. Int., vol. 35, pp. 1647-1650, 2009.

[8] T. J. Webster, C. Ergun, R. H. Doremus, R. W. Siegel, and R. Bizios, "Enhanced osteoclast-like cell functions on nanophase ceramics," Biomaterials, vol. 22, pp. 1327-1333, 2001.

[9] N. Rameshbabu, K. Prasad Rao, and T. S. S. Kumar, "Accelerated microwave synthesis of nanocrystalline hydroxyapatite," J. Mater. Sci., vol. 40, pp. 6319-6323, 2005.

[10] N. Rameshbabu, T. S. S. Kumar, and K. P. Rao, "Synthesis of nanocrystalline fluoridated hydroxyapatite by microwave processing and its in vitro dissolution study," Bull. Mater. Sci., vol. 29, pp. 611-615, 2006.

[11] S. K. Garg, "Environmental engineering," Water Supply Engineering, Khanna Publishing, vol. 1, 1992.

[12] K. Venkateswarlu, A. C. Bose, and N. Rameshbabu, "X-ray peak broadening studies of nanocrystalline hydroxyapatite by Williamson-Hall analysis," Physica B, vol. 405, pp. 4256-4261, 2010.

[13] C. Suryanarayana and M. G. Norton, X-ray diffraction: A practical approach, New York: Plenum Press Publishing, 1998.

[14] R. Yogamalar, R. Srinivasan, A. Vinu, K. Ariga, and A. C. Bose, "X-ray peak broadening analysis in ZnO nanoparticles," Solid State Commun., vol. 149, pp. 1919-1923, 2009.

[15] D. E. Gray, American institute of physics handbook, New York: McGraw-Hill Book Company, 1972. 
[16] T. Mahalingam, V. S. John, and L. S. Hsu, "Microstructural analysis of electrodeposited zinc oxide thin films," J. New Mater. Electrochem. Syst., vol. 10, pp. 9-14, 2007

[17] H. M. Burt and A. G. Mitchell, "Crystal defects and dissolution," Int. J. Pharm., vol. 9, pp. 137-152, 1981.

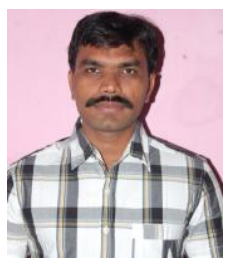

K. Venkateswarlu obtained his M.Tech. degree (2009) from the Department of Metallurgical and Materials Engineering and presently is pursuing Ph.D. degree in the Department of Physics, National Institute of Technology, Tiruchirappalli, Tamil Nadu, India. His research interests are surface modification and characterization of biomaterials.

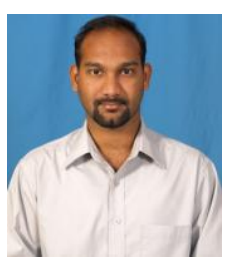

D. Sreekanth obtained his M.Tech. degree in 2005 and presently pursuing Ph.D. degree in the Department of Metallurgical and Materials Engineering, National Institute of Technology, Tiruchirappalli, Tamil Nadu, India. His research interest is surface modification of biodegradable implant materials.

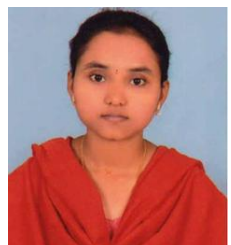

M. Sandhyarani obtained her M.Tech. degree in 2009 and presently pursuing Ph.D. degree in the Department of Metallurgical and Materials Engineering, National Institute of Technology, Tiruchirappalli, Tamil Nadu, India. Her research interests are plasma electrolytic oxidation treatment and corrosion study of biomedical implant materials.

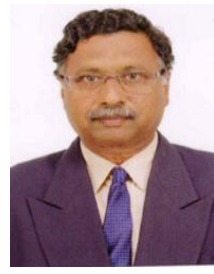

V. Muthupandi is a doctorate from Indian Institute of Technology, Madras, India. His research interests are structure property correlation, plasma electrolytic oxidation and corrosion engineering.

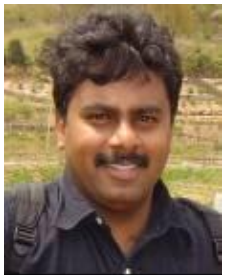

A. Chandra Bose is currently working as associate professor in the Department of Physics, National Institute of Technology, Tiruchirappalli, India. His current research interests are in the field of oxide nanomaterials and microplasma for MEMS applications.

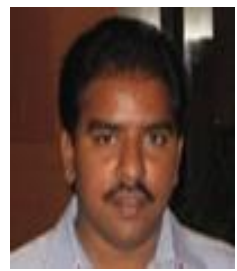

N. Ramesh Babu is currently working as assistant professor in the Department of Metallurgical and Materials Engineering, National Institute of Technology, Tiruchirappalli, India. His research interests are in the field of advanced bioceramics, surface modification of metallic implants, nanostructured processing of materials. 